\title{
La Violencia de Género como causa de Maltrato Infantil.
}

\author{
Gender Violence as cause of Child Abuse.
}

\section{A. Sepúlveda García de la Torre ${ }^{1}$}

\section{RESUMEN}

La violencia contra las mujeres o violencia de género está recibiendo una mayor atención social e institucional en nuestro país culminando con la Ley Orgánica 1/2004, de 28 de diciembre, de Medidas de Protección Integral contra la Violencia de Género. La exposición a la violencia de género en el ámbito doméstico se ha demostrado que es también causante de efectos negativos para los hijos e hijas de las mujeres víctimas, cualquiera que sea la edad de los menores, quienes pueden sufrir la violencia de forma directa, en forma de malos tratos físicos o emocionales, o de forma indirecta, siendo testigos de los actos violentos que padecen sus madres. En el presente trabajo se exponen las repercusiones que tiene para el desarrollo evolutivo, emocional, cognitivo y social de los menores, el ser testigos de la violencia hacia sus madres, así como el problema de la transmisión transgeneracional de los comportamientos violentos.

Palabras clave: Violencia familiar, violencia de género, maltrato infantil.

Cuad Med Forense 2006; 12(43-44):149-164

\section{ABSTRACT}

Violence against women or gender-based violence is receiving more social and institutional attention in Spain, culminating in the Organic Law I/2004, December 28th, of Integral Protection Measures Against Gender-based Violence. It has been demonstrated that the exhibition to gender-based violence in the domestic area is a cause of negative effects on sons and daughters of female victims as well, whatever the age of children, who can suffer the violence in a direct way, through physical or psychical abuse, or indirectly, being witnesses of violent acts whose mothers are victims. In the present work, we exposed the repercussions on evolutionary, emotional, cognitive and social development of children by being witnesses of violence towards their mothers, as well as the intergenerational transmission of violent behaviours.

Key words: Family violence, Gender violence, child abuse.

Correspondencia: Centro Asistencia a Víctimas AMUVI. c/ Alberto Lista n 16. 41003 Sevilla. Tfno.: 954905 649. Fax: 954903146. E-mail: angelessepulveda@angelessepulveda.e.telefonica.net .

1 Especialista en medicina legal y forense. Experta en mediación familiar. 


\section{INTRODUCCIÓN:}

La violencia de género es una de las formas de violencia que más atención social e institucional ha recibido en nuestro país en los últimos años, culminando entre otros, con medidas legislativas como la aprobación de la Ley 27/2003, de 31 de julio, reguladora de la Orden de Protección de las Víctimas de Violencia Doméstica, y la Ley Orgánica I/2004, de 28 de diciembre, de Medidas de Protección Integral contra la Violencia de Género; así como del II Plan Nacional de Medidas contra la Violencia Doméstica 200 I/2004, en el marco del Observatorio Nacional de la Violencia Doméstica y de Género.

En los últimos años se han realizado muchos y diversos trabajos científicos acerca de las consecuencias de la violencia de género desde el punto de vista jurídico, social, médico o psicológico; y en general sobre las repercusiones de la violencia doméstica sobre las mujeres. Sin embargo, apenas hay estudios que traten de los efectos que esta violencia tiene en las hijas e hijos de las mujeres víctimas, a pesar de que es unánime la opinión de los expertos de que los menores son también víctimas del maltrato a sus madres, y de que la violencia contra la mujer suele ir acompañada de violencia contra los menores, perpetuándose así un ciclo de comportamientos destructivos y modelos de conductas negativas en el niño que crece en presencia de esos episodios.

Es una trágica paradoja que las mujeres y la infancia corran mayores riesgos allí donde deberían disfrutar de mayor seguridad: en su propio hogar. En este sentido, nuestra Ley Orgánica de Medidas de Protección Integral contra la Violencia de Género, reconoce en su Exposición de Motivos [I] que "las situaciones de violencia sobre la mujer afectan también a los menores que se encuentran dentro de su entorno familiar, víctimas directas o indirectas de esta violencia", contemplando la Ley la protección de estos menores, no solo para la tutela de sus derechos, sino también para garantizar de forma efectiva las medidas de protección adoptadas respecto de la mujer.

\section{CONCEPTUALIZACIÓN:}

La violencia doméstica y de género no pueden ser consideradas como fenómenos unitarios. Aunque es frecuente utilizar de manera indistinta los términos violencia de género y violencia doméstica, se trata de conceptos diferentes que pasamos a exponer a continuación:

La Declaración de las Naciones Unidas sobre Erradicación de la Violencia contra las Mujeres, adoptada por la Asamblea General de la ONU en 1993, proporciona un marco amplio y útil para definir la violencia contra la mujer, aunque para otros fines concretos, como puede ser el monitoreo del problema, se necesitarían definiciones operativas más específicas. En dicha Declaración, se define esta forma de violencia [2], como "cualquier acto de violencia basada en el género que produzca o pueda producir daños o sufrimientos físicos, sexuales o mentales en la mujer, incluidas las amenazas de tales actos, la coerción o la privación arbitraria de la libertad, tanto en la vida pública como en la privada". En la Declaración se dice también, que la violencia contra la mujer abarca, entre otras, "la violencia física, sexual y psicológica que se produce en el seno de la familia y en la comunidad en general, incluidas las palizas, el abuso sexual de niñas, la violencia relacionada con la dote, la violación marital, la mutilación genital femenina y otras prácticas tradicionales dañinas para la mujer, la violencia no conyugal y la violencia relacionada con la explotación, el acoso sexual y la intimidación en el trabajo, en las instituciones educativas y en cualquier otro lugar, el tráfico de mujeres, la prostitución forzada y la violencia perpetrada o tolerada por el Estado", asociando todas estas formas de violencia a desigualdades de poder entre hombres y mujeres.

De una forma más concreta, en nuestro país, la Violencia de Género también queda expuesta en Ley Orgánical/2004, de Medidas de Protección Integral contra la Violencia de Género, [I] de la 
siguiente forma: "Se trata de una violencia que se dirige sobre las mujeres por el hecho mismo de serlo, por ser consideradas, por sus agresores, carentes de los derechos mínimos de libertad, respeto y capacidad de decisión". En cuanto a los actos de violencia, el artículo I.3 de dicha Ley explicita: "La violencia de género comprende todo acto de violencia física y psicológica, incluidas las agresiones a la libertad sexual, las amenazas, las coacciones o la privación arbitraria de libertad.

Por otra parte, la Violencia Doméstica se distingue de la anterior, además del objeto de la violencia, en el contexto donde ocurre, así la violencia doméstica [3] comprende cualquier acto de violencia llevado a cabo por quienes sostienen o han sostenido un vínculo afectivo, conyugal, de pareja, paterno-filial o semejante con la victima.

El maltrato a menores en el entorno doméstico entraría dentro de este último concepto, considerado siguiendo el postulado del Centro Internacional de la Infancia de París, como aquellos actos que privan al niño o niña de sus derechos y libertades, e inciden negativamente en su bienestar y desarrollo integral, causando intenso sufrimiento a el/la menor [4], cuyos efectos negativos aumentan a medida que la situación se cronifica.

La cuestión acerca de si el maltrato a las madres se debe incluir como una forma de maltrato infantil, ha dado lugar a debates, si bien son mayoría quienes mantienen una respuesta afirmativa, y es porque [5] gran parte de los problemas que se generan en el desarrollo de estos niños y niñas, tienen su origen, bien en las situaciones de tensión, negligencia o abandono a las que se ven sometidos por parte de sus progenitores, incapaces de satisfacer sus necesidades básicas en el clima familiar violento, bien porque son también víctimas del maltrato activo (físico o emocional) similar al que reciben sus madres o las figuras femeninas que ejercen dicho papel. En este sentido, UNICEF [6], basándose en la Convención de Derechos del Niño, que en su Artículo 19 habla de la protección ante "cualquier forma de violencia física o mental", determina la necesidad de considerar maltrato, no solo la violencia directa, sino también los efectos indirectos de la violencia familiar sobre los niños; basándose en estudios acerca de la violencia intrafamiliar que han puesto de relieve los efectos psicológicos potencialmente adversos que tienen sobre los niños presenciar o escuchar situaciones violentas (tanto físicas como psicológicas) entre sus progenitores.

\section{MAGNITUD DEL PROBLEMA:}

El conocimiento real de la incidencia de este problema, se ve obstaculizado por el oscurantismo que tradicionalmente acompaña a la violencia familiar, que impide sacar a la luz los hechos; a esto se añade la falta de conocimiento y de detección por parte de profesionales, así como otros problemas metodológicos a la hora de realizar estudios, como es el que no exista una definición unitaria de violencia de aplicabilidad transcultural, aspecto que surge en el contexto de los estudios internacionales, y que dificulta la comparación de las cifras procedentes de cada país.

Los datos en conjunto de los diferentes estudios indican que la violencia doméstica es un problema de dimensiones importantes y generalizado, que puede encontrarse en una amplia variedad de circunstancias. Todo ello ha llevado a la Organización Mundial de la Salud, a pronunciarse acerca de que la violencia contra las mujeres y menores, es uno de los problemas de salud más importantes por su creciente incidencia y mortalidad [7]; en este sentido la $49^{a}$ Asamblea Mundial de la Salud, en su Resolución WHA49.25, reconociendo las graves consecuencias inmediatas y futuras a largo plazo que la violencia tiene para la salud y para el desarrollo psicológico y social de los individuos, las familias, las comunidades y los países, declaró que la violencia es un importante problema de salud pública en todo el mundo, e instó a los Estados Miembros que evaluaran el problema de la violencia en sus territorios, y comunicaran a dicha Organización, la información que 
dispongan sobre este problema y su manera de afrontarlo. Existe pues desde 1997 un mandato de la Unión Europea para recoger, elaborar y publicar anualmente los datos sobre la violencia contra las mujeres en cada uno de los países miembros, si bien la recogida de datos a partir de las comisarías, los juzgados y los hospitales no ha hecho más que empezar, y a pesar de las indicaciones europeas, todavía no se ha iniciado el empleo de registros unificados para todo el país.

De los diferentes estudios que se han hecho, destacamos la Encuesta de Victimización, ya clásica, pero una las más amplias que se han realizado, de Straus y Gelles en el Reino Unido, que concluyeron determinantemente en 1986 [8], que la familia es uno de los grupos sociales en los que se dan más comportamientos violentos, afirmando que es más probable que una persona sea golpeada o asesinada en su propio hogar por otro miembro de su familia, que en ningún otro sitio o por ninguna otra persona.

La revisión de un número seleccionado de encuestas de población, bien diseñadas, indica que entre el $20 \%$ y el $50 \%$ de las mujeres, notifican haber sido objeto de violencia física por parte de un compañero masculino íntimo, al menos una vez en sus vidas [7].

En nuestro país, dentro de las estadísticas de violencia intrafamiliar [9], el 77\% corresponde a violencia en la pareja (del hombre a la mujer), frente a un $8 \%$ que se sitúa la violencia contra menores, y la realizada contra ancianos u otros convivientes que supone un I 5\%; así como estimaciones del Ministerio del Interior, I/3 del total de los casos de homicidio cometidos anualmente tienen como víctima y victimario a miembros de una misma familia y alrededor de $1 / 4$ parte de las denuncias de delitos y faltas de lesiones presentadas en dependencias policiales se producen en el ámbito familiar. A las estimaciones oficiales, habría que añadir los casos que conforman la llamada cifra negra, casos de violencia física o psicológica que ocurren dentro del contexto familiar no denunciados y, por tanto, ocultos a las estadísticas, que se estiman representan entre un 10-30\% de los casos reales.

En cuanto al maltrato a la mujer en España, los únicos datos de frecuencia que se han hecho públicos son los aportados por la Macroencuesta realizada por el Instituto de la Mujer, realizada en 1999 [ I0], que indica una cifra del 9,2\% de mujeres consideradas "técnicamente" maltratadas y un 2, $1 \%$ que se declaran como tales. Las diferencias en estas cifras podrían señalar que un elevado porcentaje de la violencia es aceptado por las mujeres o vivido por ellas como algo "natural" en su relación, o bien que estas identifican la violencia solo con un maltrato físico. En una segunda encuesta, realizada en el año 2002, las frecuencias son del 8,7 y el 2,3\%, respectivamente (datos no publicados).

El número de denuncias según los datos estadísticos elaborados por el Instituto Andaluz de la Mujer [I I] a partir de datos del Ministerio del Interior, en España denunciaron 57.527 y 59.758 mujeres los años 2004 y 2005, respectivamente, las cuales pertenecían a la Comunidad Autónoma Andaluza I2.42I (año 2004) y 13.69I (año 2005) mujeres (Tabla I).

TABLA I:

\begin{tabular}{|c|c|c|c|c|c|}
\hline & $\mathbf{2 0 0 2}$ & $\mathbf{2 0 0 3}$ & $\mathbf{2 0 0 4}$ & $\mathbf{2 0 0 5}$ & $\begin{array}{c}\mathbf{2 0 0 6} \\
\text { (hasta agosto) }\end{array}$ \\
\hline Andalucía & 8.848 & 10.503 & 12.421 & 13.691 & 9.586 \\
\hline España & 43.313 & 50.090 & 57.527 & 59.758 & 41.649 \\
\hline
\end{tabular}

Denuncias de mujeres víctimas de violencia por parte de su pareja o expareja.

(Solo se incluyen los casos atendidos por los Cuerpos y Fuerzas de Seguridad). [11]. 
Ante estas cifras surge la pregunta, lógica, acerca de qué ocurre con los hijos e hijas de estas mujeres víctimas. Los datos de los estudios, en su conjunto, indican que los niños que viven en hogares con agresiones a la pareja, tienen entre tres y nueve veces más posibilidades de ser maltratados físicamente por sus padres [12], Corbalán y Patró [13] en un estudio realizado sobre una muestra de mujeres maltratadas residentes en centros de acogida, concluyeron que el $85 \%$ de los hijos fueron testigos de la violencia ejercida sobre sus madres, y en un 66,6\% de los casos también ellos fueron maltratados, mayoritariamente de manera física y psicológica. El estudio realizado por UNICEF y Body Shop titulado 'Tras las puertas cerradas" [1 4] revela que en España hay cerca de 188.000 menores expuestos a esta violencia en sus casas, datos que pueden extrapolarse a 275 millones de niños en el mundo, que cada año son testigos de actos violentos en su familia.

\section{EFECTOS DE LA VIOLENCIA DE GÉNERO EN LA INFANCIA:}

Uno de los mitos de la violencia de género es que el maltrato a las mujeres, no supone un riesgo para sus hijos; esta idea sin embargo choca con la realidad, puesta de manifiesto, tanto por la frecuencia con la que los comportamientos violentos de los padres maltratadores afectan a los niños y niñas de forma directa, como por los efectos negativos que sobre el desarrollo infantil y adolescente tiene la exposición a situaciones de violencia indirecta. Como se expondrá a continuación, los problemas que presentan los menores expuestos a la violencia indirecta, son superponibles al patrón descrito en menores víctimas directas de maltrato.

Siguiendo a autores como Espinosa [5], hay una serie de consecuencias comunes tanto de la exposición directa como la indirecta a la violencia, tanto que básicamente son las mismas, si bien lo que varía es el origen de estas, y por tanto, la intervención y el tratamiento que se ha de llevar a cabo para que los miembros más vulnerables de la familia puedan llegar a superar el efecto que tienen sobre el desarrollo de los/as menores, vivir en un entorno familiar nocivo. Las principales consecuencias, en la infancia y en la adolescencia, de la exposición directa e indirecta a situaciones de violencia intrafamiliar se podrían resumir del modo que se expone en la Tabla II.

Puede observarse que gran parte de los trastornos que aparecen cuando los niños y niñas están expuestos de manera directa a la violencia tienen su origen en la incapacidad de los progenitores de satisfacer las necesidades biológicas, psicológicas y emocionales de los niños y niñas [15], al igual que ocurre en el caso de la exposición indirecta, por parte tanto de quien desempeña el rol de víctima como el de agresor, cuyos efectos observados son superponibles al patrón descrito de víctima directa.

Pedreira Massa [16] describió el "Círculo Interactivo de la Violencia Familiar", basándose entre otros, en los trabajos de Wolfe y de Paul Breitner, quien exponía que el inicio de la violencia familiar acontece con la violencia de género, que luego suele generalizarse al conjunto de los integrantes de la familia; este autor exponía que los malos tratos a la infancia están ligados a la consideración que esa sociedad posee de las mujeres, para lo que se basó en una comparación transcultural e histórica en sociedades como la judía, la mahometana, la china y la cristiano-occidental.

En la teoría del Circulo Interactivo de la Violencia Familiar, (Fig. I) la mujer víctima, como consecuencia del maltrato por parte de su pareja, padece una alta tasa de estrés, lo que puede originar, por una parte un proceso vincular con su hijo/a donde a su vez predomina el estrés, y además padecer síntomas psicológicos y físicos (angustia, trastornos depresivos, trastornos 
somatomorfos, descompensaciones de procesos crónicos como diabetes o hipertensión arterial), derivados de dicha situación, y que puede llevar a una reducción en sus habilidades de manejo eficaz de los hijos/as. Una segunda consecuencia del maltrato en la mujer es que los hijos/as padecen también una situación de estrés, fundamentalmente referido a la respuesta al proceso vincular, y al establecimiento y desarrollo de las conductas de apego, incrementándose en los menores los síntomas emocionales (irritabilidad, trastornos afectivos tipo reacciones depresivas, trastornos somatoformes) y comportamentales (descargas agresivas, oposicionismo). La alteración del proceso vincular afectado va a potenciar en el polo materno un incremento en la tensión a la hora de poder establecer la marentalidad y en el polo filial también se constata una tensión creciente en las relaciones madre-hijo, lo que sin duda repercute negativamente en el estado emocional de ambos.

TABLA II:

\begin{tabular}{|c|c|}
\hline $\begin{array}{c}\text { TIPO DE EXPOSICIÓN A LA } \\
\text { VIOLENCIA }\end{array}$ & PRINCIPALES CONSECUENCIAS \\
\hline $\begin{array}{l}\text { DIRECTA: } \\
\text { Los/as menores experimentan el } \\
\text { mismo tipo de actos violentos que la } \\
\text { madre. } \\
\text { Insultos, amenazas, humillaciones, } \\
\text { desvalorización. } \\
\text { Manipulación emocional, } \\
\text { intimidación. } \\
\text { Maltrato físico activo (bofetones, } \\
\text { patadas, palizas...) }\end{array}$ & $\begin{array}{l}\text { Consecuencias físicas (exceptuando } \\
\text { lesiones): retraso en el crecimiento, } \\
\text { alteraciones del sueño y de la alimentación, } \\
\text { retraso en el desarrollo motor, etc. } \\
\text { Alteraciones emocionales: ansiedad, } \\
\text { depresión, baja autoestima, trastorno de estrés } \\
\text { post-traumático, etc. } \\
\text { Problemas cognitivos: retraso en el lenguaje, } \\
\text { absentismo escolar, fracaso escolar, etc. } \\
\text { Problemas de conducta: falta de habilidades } \\
\text { sociales, agresividad, inmadurez, delincuencia, } \\
\text { toxicomanías, etc. }\end{array}$ \\
\hline $\begin{array}{l}\text { INDIRECTA: } \\
\text { Se considera cuando la agresión } \\
\text { ocurre en su presencia, y también } \\
\text { aún cuando no estén presentes, pero } \\
\text { no pueden tomar distancia de sus } \\
\text { progenitores. } \\
\text { Oyen disputas, golpes, perciben } \\
\text { terror. }\end{array}$ & $\begin{array}{l}\text { Incapacidad de las víctimas (las madres) } \\
\text { para atender a las necesidades básicas de las } \\
\text { niñas y niños, por la situación física y } \\
\text { emocional en la que se encuentran. Lo que } \\
\text { puede generar situaciones de negligencia y } \\
\text { abandono hacia los niños y niñas. } \\
\text { Incapacidad de los agresores de establecer } \\
\text { una relación cálida y afectuosa cercana con } \\
\text { sus hijas e hijos. Lo que puede generar serios } \\
\text { problemas de vinculación afectiva y } \\
\text { establecimiento de relaciones de apego. }\end{array}$ \\
\hline
\end{tabular}

Principales consecuencias de la exposición directa o indirecta a situaciones de violencia intrafamiliar. Adaptada de [5]. 


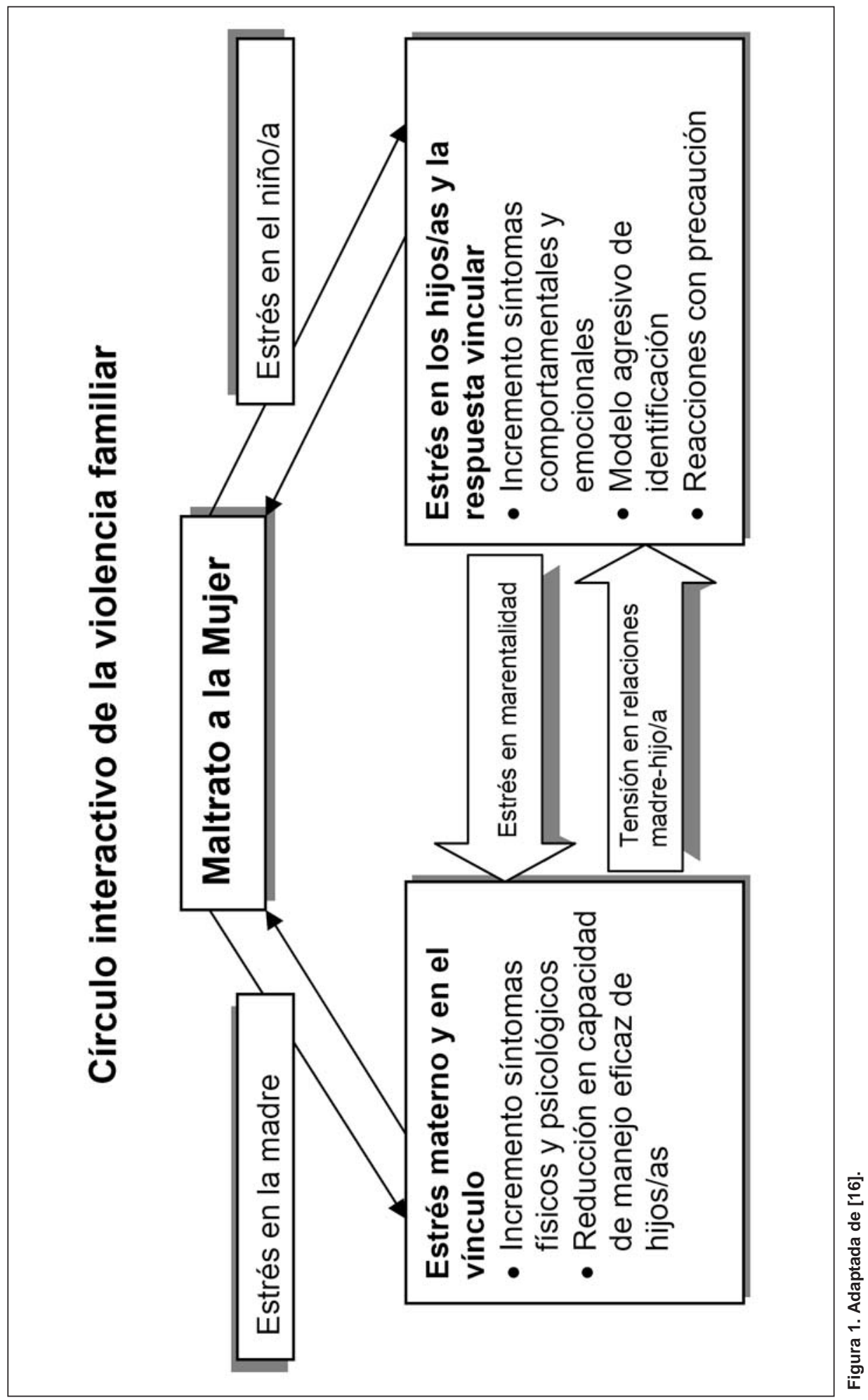


Esta situación de estrés de la mujer víctima no debe en ningún caso ser culpabilizadora para ella ni convertirla en blanco de las críticas. No todas las mujeres reaccionan igual, y la mayoría de las veces, la toma de conciencia de que el maltrato que ellas sufren le está afectando a sus hijos, les da fuerza para romper el círculo en el que se ven inmersas.

De una manera más precisa la Tabla III resume la interacción entre las conductas de agresión de la figura maltratadora, el impacto en el otro polo de la pareja maltratada y la repercusión en los hijos e hijas de esta situación de violencia familiar.

TABLA III:

\begin{tabular}{|c|c|c|}
\hline MALTRATADOR & PAREJA MALTRATADA & HIJOS E HIJAS \\
\hline $\begin{array}{l}\text { EL MALTRATADOR SE } \\
\text { CARACTERIZA POR: }\end{array}$ & $\begin{array}{c}\text { LA PAREJA MALTRATADA SE } \\
\text { CARACTERIZA POR: }\end{array}$ & $\begin{array}{c}\text { LOS/AS HIJOS/AS QUE } \\
\text { CONVIVEN EN HOGARES CON } \\
\text { MALOS TRATOS SE } \\
\text { CARACTERIZAN POR: }\end{array}$ \\
\hline $\begin{array}{l}\text { Dificultad en el control de los } \\
\text { impulsos (escasa tolerancia a la } \\
\text { frustración, temperamento } \\
\text { explosivo). } \\
\text { Rabia. } \\
\text { Precisa de "pruebas" continuas } \\
\text { de afecto. }\end{array}$ & $\begin{array}{l}\text { Mucho tiempo ser-suficiente. } \\
\text { Tiempo de frustración. } \\
\text { Aceptación pasiva. } \\
\text { Angustia. }\end{array}$ & $\begin{array}{l}\text { Combinación de limitada } \\
\text { tolerancia a la frustración, } \\
\text { control pobre de impulsos y } \\
\text { mucho tiempo frustrado/a. } \\
\text { Angustia que se manifiesta hacia } \\
\text { el interior-exterior. }\end{array}$ \\
\hline $\begin{array}{l}\text { Trastornos por ansiedad. } \\
\text { Funcionamiento psicosomático. } \\
\text { Dependencia emocional. } \\
\text { Sintomas sofisticados que } \\
\text { aparecen como disfunciones en } \\
\text { los niveles socio-educativo. } \\
\text { Depresiones sólo en el ámbito } \\
\text { familiar. }\end{array}$ & $\begin{array}{l}\text { Trastornos por ansiedad. } \\
\text { Funcionamiento psicosomático. } \\
\text { Dependencia emocional y a } \\
\text { veces económica. } \\
\text { Riesgo de consumo secreto de } \\
\text { drogas y alcohol. } \\
\text { Riesgo de accidentes } \\
\text { domiciliarios. }\end{array}$ & $\begin{array}{l}\text { Tristeza, depresión. } \\
\text { Trastornos por ansiedad. } \\
\text { Funcionamiento psicosomático. } \\
\text { Absentismo escolar. } \\
\text { Conductas predelincuente y } \\
\text { delincuente. } \\
\text { Dependencia económica y } \\
\text { emocional. } \\
\text { Alto riesgo de consumo de } \\
\text { drogas y alcohol. } \\
\text { Aislamiento, soledad. } \\
\text { Fugas, miedo. }\end{array}$ \\
\hline $\begin{array}{l}\text { Limitación para solicitar ayudas } \\
\text { salvo las muy orientadas al } \\
\text { presente inmediato. }\end{array}$ & $\begin{array}{l}\text { Paciencia ilimitada para buscar } \\
\text { la "poción mágica" de solución } \\
\text { de problemas. }\end{array}$ & $\begin{array}{l}\text { Combinación de deficiente } \\
\text { control de impulsos y } \\
\text { permanente optimismo ante las } \\
\text { nuevas situaciones. }\end{array}$ \\
\hline $\begin{array}{l}\text { Necesidades yoicas insaciables } \\
\text { con aspectos de narcisismo } \\
\text { infantil (no detectables fuera del } \\
\text { grupo familiar). } \\
\text { Cualidades que sugieren gran } \\
\text { potencial de cambio y mejoria } \\
\text { (promesas, cambios de futuro). }\end{array}$ & $\begin{array}{l}\text { Inseguridad en conocer las } \\
\text { propias necesidades que se } \\
\text { presentan en función de pareja, } \\
\text { familia, trabajo... } \\
\text { Esperanza irreal en que el } \\
\text { cambio es inminente. } \\
\text { Creencia en promesas. }\end{array}$ & $\begin{array}{l}\text { Inestable definición del yo. } \\
\text { Envidia con caprichos infantiles. } \\
\text { Deseos en base a los parentales. } \\
\text { Mezcla de esperanza/depresión } \\
\text { en que va a cambiar. }\end{array}$ \\
\hline $\begin{array}{l}\text { Percepción de pobres } \\
\text { intercambios sociales. } \\
\text { Relación de pareja cerrada. } \\
\text { Exclusión de contactos fuera de } \\
\text { la familia. }\end{array}$ & $\begin{array}{l}\text { Incremento gradual del } \\
\text { aislamiento social. } \\
\text { Escasos contactos fuera de la } \\
\text { familia. }\end{array}$ & $\begin{array}{l}\text { Incremento del aislamiento } \\
\text { social. } \\
\text { Aislamiento con padres, } \\
\text { identificación con ellos. } \\
\text { Pobreza en habilidades sociales. }\end{array}$ \\
\hline
\end{tabular}


TABLA III CONTINUACIÓN:

\begin{tabular}{|c|c|c|}
\hline MALTRATADOR & PAREJA MALTRATADA & HIJOS E HIJAS \\
\hline $\begin{array}{l}\text { EL MALTRATADOR SE } \\
\text { CARACTERIZA POR: }\end{array}$ & $\begin{array}{l}\text { LA PAREJA MALTRATADA SE } \\
\text { CARACTERIZA POR: }\end{array}$ & $\begin{array}{l}\text { LOS/AS HIJOS/AS QUE } \\
\text { CONVIVEN EN HOGARES CON } \\
\text { MALOS TRATOS SE } \\
\text { CARACTERIZAN POR: }\end{array}$ \\
\hline $\begin{array}{l}\text { Acusaciones a la pareja. } \\
\text { Celos. Miedo al abandono. } \\
\text { Posesivo. Controlador. }\end{array}$ & $\begin{array}{l}\text { Inhabilidad para convencer al } \\
\text { compañero de su lealtad. } \\
\text { Escasa ayuda y poder en la } \\
\text { pareja. }\end{array}$ & $\begin{array}{l}\text { Conductas disruptivas con los } \\
\text { padres. } \\
\text { Intercambios afectivos pobres. }\end{array}$ \\
\hline Miedo a estar solo. & $\begin{array}{l}\text { Miedo constante que se va } \\
\text { haciendo acumulativo y opresor } \\
\text { con el tiempo. }\end{array}$ & $\begin{array}{l}\text { Miedo y terror en la vida y por } \\
\text { sus padres. } \\
\text { Confusión e inseguridad. }\end{array}$ \\
\hline $\begin{array}{l}\text { Violación de los límites de los } \\
\text { otros: espiar, etc. } \\
\text { Culpar a otros de sus propios } \\
\text { actos. }\end{array}$ & $\begin{array}{l}\text { Perdida gradual de los propios } \\
\text { limites. } \\
\text { Aceptación de todas las culpas. }\end{array}$ & $\begin{array}{l}\text { Pobre definición de los limites } \\
\text { personales. } \\
\text { Violación de los limites de los } \\
\text { demás. }\end{array}$ \\
\hline
\end{tabular}

Características comportamentales en los malos tratos: interacción entre maltratador-pareja maltratadahijos/as. Adaptada de [16].

\section{EL IMPACTO DE LA VICTIMIZACIÓN:}

Las repercusiones psicológicas van a depender de varios factores:

- Las características personales de la víctima (edad, desarrollo, vulnerabilidad, si padece alguna discapacidad).

- Las circunstancias del maltrato (cronicidad, tipología, gravedad, severidad de este, así no es lo mismo presenciar amenazas, que un parricidio).

- La protección que ejerza la figura materna y el entorno familiar sobre el/la menor.

Para valorar el impacto, nos basamos en los postulados de Finkelhor sobre la Victimología Evolutiva, entendiendo por tal el estudio de la diversidad de victimizaciones que los niños pueden experimentar a lo largo de las diversas fases de la infancia [17], conocemos que la manera en que los niños y niñas responden a la victimización, depende de su vulnerabilidad y de las capacidades específicas de su estadío de desarrollo, y dentro de estas la valoración que el/la menor hace sobre la victimización, y las estrategias de afrontamiento que hace ante ellas, pudiendo desempeñar espontáneamente el papel de pararrayos tratando de desviar la atención de sus padres para distraer su agresividad, y así ponen en marcha distintos modos de agitación y defensa, o bien utilizan un caparazón o refugiándose en un mundo imaginario.

Según las diferentes etapas evolutivas [16,18,19,20,21] podemos exponer algunos de los problemas de ser testigos de violencia, es importante recordar que los niños y niñas no expresan sus pensamientos y emociones verbalmente sino más bien con el comportamiento; el grado en que pueden verbalizar sus pensamientos depende de su grado de desarrollo, de sus características de personalidad y del tipo de relación familiar y social :

Ya en el embarazo la madre puede sufrir malos tratos físico o psicológicos, de hecho en esta etapa hay veces que marca el principio de la violencia o esta se recrudece. Las consecuencias pueden ser parto prematuro, bajo peso al nacer, mortalidad perinatal; también se tiene conocimiento 
que las mujeres en situación de malos tratos, tienen menos seguimiento del embarazo, hay más interrupciones voluntarias del mismo, y participan menos en la preparación para el parto. Además hay más posibilidades de consumo de alcohol y ansiolíticos por parte de la madre.

Las consecuencias psicológicas durante la primera infancia y la edad preescolar están relacionadas con el desarrollo del apego. Los niños que experimentan malos tratos pueden crecer con una falta o una desorganización en el apego, se observan además trastornos de relación con sus iguales, conductas de retraimiento y retrasos cognitivos.

En la primera etapa, los/as menores perciben el miedo y la ansiedad de sus madres, en esta época pueden ser ignoradas sus necesidades llevando a la desconfianza y al abandono emocional. En la etapa preescolar (2-5 años), los/as menores observan la realidad sin comprenderla, a lo que se añade la dificultad para diferenciar la fantasía de la realidad, pueden creer que son ellos la razón del conflicto, se culpan y tienen sentimientos de inutilidad y ansiedad. La sintomatología principal en esta etapa comprenderá miedo, se sienten desamparados e impotentes y creen que pueden morir durante las agresiones, ansiedad, inseguridad, dudas, expectación, actitudes de negación y de regresión, tristeza y aislamiento.

En la infancia media (6-II años), los problemas afectan fundamentalmente al desarrollo socio-emocional. En la etapa escolar (6 a 8 años), las dificultades de comprensión y asimilación de los problemas se expresa a través de sintomatología de ansiedad, depresión y cognición (fantasías), a medida que el niño crece, aumenta su capacidad para comprender y asimilar la realidad; se puede presentar alianza con uno de los progenitores, culpabilización del otro, enojo, hostilidad, etc., lo que sienta las bases de la identificación de roles. La sintomatología ansiosa y depresiva es cuando se hace más presente, junto con el aislamiento en el entorno escolar y social para mantener en secreto "su problema". En pre-adolescentes, los sentimientos de frustración y desamparo se pueden traducir a violencia o comportamiento antisocial y mala conducta en la escuela, algunos son provocadores y agresivos para adquirir sensación de poder, otros son evitadores de relaciones. En otros casos pueden adoptar posiciones prematuramente adultas de protección a sus madres y hermanos/as (hasta los 10-12 años), pero a medida que crecen puede aumentar el desapego, embotamiento y bloqueo.

En la adolescencia se alcanza el desarrollo intelectual, pero existe un desarrollo asimétrico dado que la mayor capacidad de análisis y evaluación no va paralela a los conocimientos y experiencias que presenta el sujeto adulto, por lo que se pueden idear soluciones utópicas, presentar salidas en falso, etc. Además es una época en la que de por sí se abordan temáticas propias del ámbito de personalidad, como es la identidad personal, rol sexual, competencia personal, planteamiento de futuro, intereses, valores, conducta, etc, por lo que la vivencia del maltrato a su madre puede suponer un punto importante de desequilibrio en su desarrollo integral. En los adolescentes puede existir un fuerte sentimiento de desamparo al no poder salvar a las madres, los síntomas más frecuentes en esta etapa están relacionados con actitudes de responsabilidad excesiva en el hogar, un hecho que ocurre en esta etapa es cuando los niños adquieren un papel protector activo, de tal manera, que se interponen delante de la madre cuando el padre intenta golpearla, recibiendo ellos mismos los golpes, e incluso llegando ellos mismos a agredir a sus propios padres, a veces se han dado caso incluso de parricidios. También encontramos niveles bajos de autoestima, niveles altos de ansiedad y depresión y problemas académicos. Otras veces crean situaciones con premeditación para hacerse sentir necesitados, buscan ser aceptados. También pueden adoptar cambios radicales de estilo de vida, se escapan mediante el sexo o delincuencia. En ocasiones llegan al embotamiento emocional, frialdad e indiferencia.

De una forma general basándonos en la Victimología Evolutiva, se puede decir que los efectos de ser testigo de violencia son característicos de las etapas de la Psicopatología del 
Desarrollo, siendo propensas las víctimas a presentar problemas en su desarrollo evolutivo, emocional, cognitivo y social, que le impide el bienestar y un progreso adecuado como persona.

Los primeros estudios sobre violencia en el matrimonio realizados por Jaffe, Wilson y Wolfe en 1986 [12], pusieron de relieve la relación existente entre formas intensas de conflicto matrimonial y problemas de conducta en los hijos, encontrando más problemas externos de conducta y una menor competencia social, de hecho entre el $25 \%$ y el $70 \%$ de los niños con familias en las que se producían agresiones presentaban problemas clínicos de conductas. Otros estudios que se han realizado han mostrado que los niños expuestos a la violencia en las familias presentan más problemas externalizantes de conducta (agresividad y antisociales) e internalizantes (inhibición y miedo), que los niños que no están expuestos a la violencia familiar. Corbalán y Patró [I 3], realizaron un estudio en el 2003 con una muestra de 40 mujeres maltratadas residentes en centros de acogida, sobre los comportamientos problemáticos apreciados en sus hijos, concluyendo que los comportamientos más frecuentes eran la violencia hacia iguales (35\%) seguida del comportamiento violento a la propia madre (22\%), y en menor medida, la conflictividad en la escuela (10\%); otros síntomas informados fueron ansiedad (32\%), tristeza y aislamiento (27,5\%), miedo al maltratador $(27,5 \%)$ y bajo rendimiento escolar.

Las principales consecuencias para el desarrollo de las niñas y niños testigos de la violencia familiar se exponen en la Tabla IV.

TABLA IV:

\begin{tabular}{|c|c|}
\hline \multicolumn{2}{|r|}{ PRINCIPALES CONSECUENCIAS } \\
\hline $\begin{array}{l}\text { DESARROLLO } \\
\text { SOCIAL }\end{array}$ & $\begin{array}{l}\text { - Dificultades de interacción social. } \\
\text { - Conductas externalizantes: Problemas de agresividad. } \\
\text { - Conductas internalizantes: Problemas de inhibición y miedo. } \\
\text { - Dificultades para interpretar las claves sociales. } \\
\text { - Falta de habilidades de resolución de problemas sociales. Falta de } \\
\text { habilidad resolución de conflictos. } \\
\text { - Tendencia a interpretar de forma hostil la conducta de los otros. } \\
\text { - Aislamiento y soledad. Inseguridad, desconlianza. Falta de integración. } \\
\text { - Conductas antisociales. Delincuencia. }\end{array}$ \\
\hline $\begin{array}{l}\text { DESARROLLO } \\
\text { EMOCIONAL }\end{array}$ & $\begin{array}{l}\text { - Falta de empatía. Dificultades para expresar y comprender emociones, } \\
\text { tanto propias como ajenas. Ansiedad. Tristeza. } \\
\text { - Internalización de roles que no le corresponden a su edad } \\
\text { (paternalización y de género). } \\
\text { - Problemas de autocontrol de la propia conducta. Escasa tolerancia a la } \\
\text { frustración. Fxplosiones. }\end{array}$ \\
\hline $\begin{array}{l}\text { DESARROLlLo } \\
\text { COGNITIVO }\end{array}$ & $\begin{array}{l}\text { - Baja autoestima. } \\
\text { - Indefensión aprendida. } \\
\text { - Tendencia a no cnfrentarse a nuevas tarcas por miedo al fracaso y/o a la } \\
\text { frustración. } \\
\text { - Problemas de egocentrismo cognitivo y social. } \\
\text { - Juicios morales heterónomos: más permisivos con sus transgresiones que } \\
\text { con las de los demás. Legitimidad en el uso de la violencia. Mucha } \\
\text { pasividad o demasiada intransigencia por parte de las chicas a los chicos. } \\
\text { - Problemas en el desarrollo de la atención, memoria, concentración. }\end{array}$ \\
\hline
\end{tabular}

Principales consecuencias para el desarrollo de las niñas y niños testigos de la violencia familiar. Adaptado de [5]. 


\section{El trastorno de Estrés Postraumático en niños/as testigos de violencia a sus madres.}

Las situaciones de violencia familiar de las que los/as menores son testigo, pueden dar lugar a situaciones traumáticas crónicas, a situaciones traumáticas crónicas con fases de exacerbación y escaso control, e incluso a situaciones de presentación aguda e incontrolable (se han llegado a producir graves lesiones e incluso homicidios en presencia de los hijos), que coinciden con las tipologías II, III y I de Terr, con tan graves consecuencias para la salud mental que desencadenan un cuadro de Trastorno de Estrés Postaumático.

Tras la experiencia traumática se produce pérdida del sentimiento de invulnerabilidad, sentimiento bajo el cual funcionan la mayoría de los individuos y que constituye un componente de vital importancia para evitar que las personas se consuman y paralicen con el miedo a su propia vulnerabilidad; en el caso de los niños que no solo son testigos del maltrato hacia su madre sino que, a la vez, también son víctimas de esa violencia, la pérdida es todavía, si cabe, mucho más desequilibrante, pues afecta a un componente absolutamente necesario para el adecuado desarrollo de la personalidad del menor: el sentimiento de seguridad y de confianza en el mundo y en las personas que lo rodean [13]. Este hecho reviste especial severidad cuando el agresor es su propio padre, figura central y de referencia para el niño, y la violencia ocurre dentro de su propio hogar, lugar de refugio y protección; ya que se produce la destrucción de las bases de su seguridad, quedando el/la menor a merced de sentimientos como la indefensión, el miedo o la preocupación ante la posibilidad de que la experiencia traumática pueda repetirse, todo lo cual se asocia a una ansiedad que puede llegar a ser paralizante. Tristemente, en el caso de la violencia familiar, la experiencia temida se repite de forma intermitente a lo largo de muchos años, constituyendo una amenaza continua y muchas veces percibida como incontrolable.

Según el Manual Diagnóstico y Estadístico de los Trastornos Mentales DSM-IV [22], el Trastorno de Estrés Postraumático aparece cuando la víctima ha sufrido o ha sido testigo de una amenaza para la vida, de uno mismo o de otra persona, y reacciona con miedo, horror e indefensión; los tres aspectos nucleares de este cuadro clínico son: la víctima revive la experiencia en forma de pesadillas, imágenes, y recuerdos frecuentes e involuntarios (criterio de reexperimentación); la víctima intenta evitar o huir de lugares o situaciones relacionadas con el hecho traumático, e incluso rechazan pensar o hablar de este (criterio de evitación), y por último las víctimas muestran una respuesta de sobresalto exagerada que se manifiesta en dificultades de concentración, insomnio e irritabilidad (criterio de activación).

En los niños, la respuesta de temor pueden expresarse en comportamientos desestructurados o agitados, la reexperimentación se puede poner de manifiesto en juegos repetitivos donde aparecen temas o aspectos característicos del trauma, o sueños terroríficos de contenido irreconocible; los niños pequeños pueden reescenificar el acontecimiento traumático específico. La evitación en niños pequeños puede ser difícil de apreciar (el expresar la disminución del interés por las actividades importantes y el embotamiento de sus sentimientos y afectos, estos síntomas deben ser objeto de una cuidadosa valoración mediante el testimonio de los padres, profesores y otros observadores); en los niños la sensación de un futuro desolador puede traducirse en la creencia de que su vida no durará tanto como para llegar a adulto. También puede producirse la "elaboración de profecías", es decir, la creencia en una especial capacidad para pronosticar futuros acontecimientos desagradables. Respecto al aumento de la activación los niños pueden presentar varios síntomas físicos: dolores de estómago, de cabeza, y otros síntomas.

En la investigación realizada en el Registro Acumulativo de Casos Psiquiátricos (RACP), Pedreira [16], detectó que en casi el 60\% de los casos de menores estaba presente la violencia 
familiar, directa o como testigos, En todos los casos aparecía el diagnóstico de Trastorno de Estrés Postraumático, y destacan síntomas y signos inespecíficos (Trastornos del comportamiento, inhibición, Trastornos. del sueño, ánimo depresivo, Trastornos esfinterianos y exceso de apego a la figura materna, como más representativos), pero se acompañaba de otra serie de trastornos mentales que de forma co-mórbida evolutivamente aparecían, fundamentalmente la aparición de importantes reacciones depresivas y trastornos no orgánicos del sueño.

Margolin y Gordis [23] describen 4 conductas típicas de Síndrome de Estrés Postraumático en Niños: recuerdos repetidos de las situaciones a través de la visualización, conductas y juegos repetitivos relacionados con acontecimientos estresantes, actitudes pesimistas relacionadas con indefensión y futuro ante la vida, activación excesiva con hiperactividad y problemas de atención; estas conductas se hacen disfuncionales cuando se cronifican.

\section{Transmisión Transgeneracional de la Violencia.}

Entre los efectos a largo plazo, que se asocian a la exposición de menores a la violencia, y que son fuente de preocupación, no solo por el bienestar y desarrollo de las propias víctimas, sino por la repercusión social que tienen, se encuentra el aprendizaje que hacen los menores de las conductas violentas dentro de su hogar.

Una revisión de las investigaciones de la Academia de la Ciencia de los Estados Unidos, afirma que "la tercera parte de los niños que sufrieron abusos o se vieron expuestos a la violencia paterna, se convierten en adultos violentos" [24]. Efectivamente, los/as menores aprenden a definirse, a entender el mundo, y a relacionarse con él, a partir de lo que observan en su entorno más próximo, y en este sentido, la familia es el agente socializador más importante. Los niños que crecen en hogares violentos aprenden e interiorizan una serie de creencias y valores negativos entre los que se encuentran los estereotipos de género, desigualdades entre hombre/mujer, las relaciones con los demás, así como sobre la legitimidad del uso de la violencia como medio de resolver conflictos, que sientan las bases de comportamientos maltratantes futuros en las relaciones de pareja.

La mayoría de los autores, sostienen que la tendencia observada es que las niñas se identifiquen con el rol materno, adoptando conductas de sumisión, pasividad y obediencia; y los niños con el rol paterno, adoptando posiciones de poder y privilegio. Al fin y al cabo lo expuesto, no es más que la expresión de la socialización diferencial de género, un factor que actúa en el origen y mantenimiento de la violencia contra las mujeres, y que se trasmite no solo intrafamiliarmente, sino a través de toda la sociedad.

Salas Bahamón [25] en su estudio realizado en Colombia acerca de los mecanismos de reproducción de la violencia en 2.295 mujeres, siguiendo la metodología propuesta por Pollak (2002), en la que supone que el comportamiento agresivo se transmite por aprendizaje o por imitación; por lo cual el haber sido víctima y/o testigo de violencia entre los padres es un factor de aceptación de la violencia entre las parejas, y por lo tanto convierte a los hombres en perpetradores de maltrato y a las mujeres en sus víctimas; en esta metodología admite el divorcio como mecanismo de ruptura de la reproducción de violencia a la siguiente generación, (es decir que el divorcio es el mecanismo que más reduce el nivel de violencia). Los resultados del estudio fueron los siguientes: Las mujeres que fueron testigos de violencia entre los padres, son más propensas a unirse con hombres violentos también testigos de violencia en la familia, que las mujeres que no manifestaron haber visto violencia física severa del padre hacia la madre, $37 \%$ y $27 \%$ respectivamente. Adicionalmente, 
la proporción de mujeres que no "rompen" el ciclo de violencia, es decir que no se divorcian o permanecen con sus compañeros violentos es mayor cuando experimentaron altos niveles de violencia entre sus padres durante su infancia (63.3\%); mientras que el $60 \%$ de las mujeres que cuando niñas no estuvieron expuestas a violencia entre sus padres, no se divorcia de su compañero violento. Este resultado indica, que no solo la exposición a la violencia en la familia por parte de las mujeres es un factor que contribuye a la transmisión de la violencia, en la siguiente generación, sino que también influye en esta transmisión la exposición a violencia entre los padres del esposo o compañero.

A menudo resulta difícil separar las causas de las consecuencias: crecer en una familia en la que la madre es objeto de abusos es una vía importante para que el ciclo de la violencia doméstica se perpetúe, sin embargo existen mecanismos que rompen el ciclo del maltrato y disminuyen la proporción de hogares que sufren de violencia intrafamiliar en la siguiente generación. Aún siendo relevante que la violencia contra las madres es trasmitida de forma vicaria a los hijos e hijas, y sin duda es un factor predictor de victimización (tanto para ser futura víctima o victimario), también es cierto que una intervención terapéutica y un buen apoyo familiar y social, son fundamentales para el sano desarrollo de los/as menores; y que los antecedentes inmediatos en la vida adulta [2I], como adaptación a la vida cotidiana, calidad de relación de pareja, autoestima, habilidades de comunicación y de resolución de problemas, y capacidad de resistencia, desempeñan un papel más importante que los antecedentes de maltrato a la infancia.

\section{ATENCIÓN A LOS HIJOS E HIJAS DE LAS MUJERES VÍCTIMAS DE VIOLENCIA DE GÉNERO:}

El estudio realizado por Save the Children [26] presentado en junio de 2006, hace un análisis de la Atención a los Niños y Niñas Víctimas de Violencia de Género en el Sistema de Protección a la Mujer, en él se concluye que los hijos e hijas de las mujeres maltratadas son también víctimas de la misma violencia, y se destaca la importancia del problema, a pesar de lo cual, los recursos y programas de atención a estos niños son muy escasos, por no decir inexistentes.

Entre las principales dificultades a la hora de atender a hijos e hijas de mujeres víctimas de violencia de género (tanto en el ámbito social, policial, sanitario y judicial), que señalan el estudio, destacamos la falta de protocolos de actuación, de personal formado y especializado en la materia, y que no siempre se realizan intervenciones ni periciales a los hijos de la mujer, en los casos de niños testigos de violencia, cuando no han sufrido la violencia directa.

El estudio hace una serie de recomendaciones de mejora y buenas prácticas para garantizar la atención de calidad, algunas de las cuales enumeramos a continuación:

- Promover circuitos de actuación que garanticen la privacidad y que eviten la victimización secundaria, fortaleciendo la coordinación entre los diferentes recursos implicados en el sistema de protección. Para conseguir un intercambio de toda la información relativa a un caso y una coordinación adecuada en cuanto a las actuaciones a llevar a cabo, es primordial que el sistema judicial se implique en las Comisiones de Seguimiento.

- Diseñar programas integrales de intervención familiar, y no en separación o disgregación de sus miembros, estableciendo canales de comunicación ágiles entre todas las partes implicadas en el proceso.

- Proporcionar a los profesionales que atienden a las mujeres víctimas de violencia de género formación específica e interdisciplinar sobre la atención a niños y niñas, con el fin de crear un trabajo en red eficaz que permita una atención óptima y un seguimiento adecuado de los casos. 
- Realizar evaluaciones psicológicas a los niños y las niñas, ya que hay menores que no presentan síntomas aparentes. Los niños y niñas rara vez piden ayuda por sí mismos/as, porque no saben o no pueden.

- Promover e incrementar los programas de tratamiento terapéutico para las mujeres, niños y niñas víctimas de violencia de género.

- Informar a los hijos e hijas desde el primer momento de la situación en la que se encuentran. Qué es lo que pasa, que sucederá, cuáles serán los pasos a seguir, cómo les afecta a ellos la situación dada, etc. Hay que proporcionarles toda la información necesaria adaptándola a su nivel de comunicación y entendimiento, y sobre todo, contar con su opinión, evitando así que se sientan excluidos del proceso, cuando ellos están inmersos en el mismo.

- Incrementar la dotación de espacios físicos adecuados para los niños y niñas en el ámbito judicial y policial en los que puedan permanecer mientras que sus madres interponen la denuncia y/o prestan declaración.

- Promover la toma de declaración a los menores por medio de personal especializado en atención a las víctimas, así como la articulación de medios para evitar que el niño o la niña tenga que declarar en la sala de juicios (video conferencias, entrevistas previamente grabadas...).

- Crear puntos de encuentro diferenciados para la intervención en casos de violencia de género. La mediación en casos de violencia no es viable.

- Establecer el régimen de visitas en base a la valoración previa de las relaciones dentro de la unidad familiar, la cual debe ser realizada por profesionales que dispongan de una formación especializada. Igualmente, para llevar a cabo esta valoración, se ha de dotar a las instituciones pertinentes de los medios económicos y humanos oportunos.

Los niños y niñas testigos de violencia de género, deben contar con el mismo nivel asistencial y especial protección judicial que cuando son víctimas directas de maltrato. Es importante reconocer a los hijos e hijas de las mujeres víctimas de violencia, también como víctimas de la misma violencia, y desarrollar los recursos necesarios para atender sus necesidades específicas, es una medida imprescindible para un cumplimiento real de los objetivos con los que nació la Ley Orgánica de Medidas de Protección Integral contra la Violencia de Género.

\section{CONCLUSIÓN:}

Uno de los mitos que hay que superar, es que la Violencia de Género sólo forma parte de los conflictos de pareja. Está demostrado que el maltrato a la mujer se extiende a sus hijos e hijas, afectando negativamente su bienestar y su desarrollo, con secuelas a largo plazo; llegando incluso a transmitirse a sucesivas generaciones. Por tanto, debe ser prioritario para las instituciones evitar esta forma de maltrato infantil mediante programas de prevención y asistencia a las víctimas.

\section{AGRADECIMIENTOS:}

A Ana Soler y Ana Balestra, eficaces Trabajadoras Sociales de AMUVI en la asistencia a mujeres y menores víctimas de violencia de género. 


\section{BIBLIOGRAFÍA:}

I. Ley Orgánica I/2004 de 28 de diciembre de Medidas de Protección Integral contra la Violencia de Género.

2. Naciones Unidas (1995). Declaración de Beijing de 1995, y Plataforma para la Acción. IV Conferencia Mundial sobre las Mujeres. Beijing, China, Madrid: Ministerio de Asuntos Sociales, Instituto de la Mujer.

3. Alberdi I, Matas, N. La violencia doméstica, informe sobre los malos tratos a mujeres en España, Fundación La Caixa, Colección Estudios Sociales $n^{0}$ 10, 2002.

4. Gómez de Terreros I: Los profesionales de la salud ante el maltrato. $2^{\mathrm{a}}$ ed. Editorial Comares. Granada, 1997.

5. Espinosa Bayal- M.A. las hijas e hijos de mujeres maltratadas: consecuencias para su desarrollo e integración escolar. Instituto Vasco de la Mujer. 2004.

6. UNICEF :Innocenti Report Card Digest $n^{0} 2$. Niños y Violencia. 1999.

7. World Report on violence and Health. Geneva: World Health Organization; 2002 http://www5.who.int/violence_injury_prevention/.

8. Straus, M: Mofogénesis social y violencia familiar desde una perspectiva transcultural. En: Honorio M. Velasco (comp). Lecturas de Antropología Social y Cultural. UNED. Madrid, 1996. Pp:713-730.

9. Cerezo Domínguez Al. Las Victimas de Violencia domestica. Manual de victimologia. Tirant lo Blanc. Valencia. 2006. Pp:164-190.

10. La violencia contra las mujeres. Resultados de la macroencuesta. Madrid: Instituto de la Mujer. Ministerio de Trabajo y Asuntos Sociales; 1999

II. Instituto Andaluz de la Mujer. Denuncias de mujeres víctimas de violencia por parte de su pareja o expareja en Andalucía y España. Publicado en internet, 2006. http://www.juntadeandalucia.es/institutodelamujer/datos_estadisticos/capitulo6.htm.

12. Cantón Duarte J, Cortes Arboleda MR, Justicia Díaz MD. Conflictos matrimoniales, divorcio y desarrollo de los hijos. $2^{\mathrm{a}}$ ed. Pirámide. Madrid. 2002. Pp:51-59.

13. Patró Hernández R, Limiñana Gras RM. Victimas de Violencia Familiar, Consecuencias Psicológicas en Hijos de Mujeres Maltratadas. Anales de Psicología 2005;21:II-17.
I4. UNICEF. Venid closed doors. The impact of domestic violence on children. Editorial Unicef. Nueva York/Londres, 2006.

15. Ochaíta E, Espinosa MA. Hacia una teoría de las necesidades infantiles y adolescentes: necesidades y Derechos en el marco de la Convención de Naciones Unidas sobre Derechos del Niño. 2004.

16. Pedreira Massa JL. La infancia en la familia con violencia: Factores de riesgo y contenidos psico(pato)lógicos. Psiquiatria.com. 2003;7(4).

17. Finkelhor D. Victimología Infantil. En: Sanmartin J (eds). Violencia contra niños. Ariel, Centro Reina Sofía para el Estudio de la Violencia. 1999. Pp:I47-171.

18. Zubizarreta Anguerra I. Consecuencias Psicológicas del Maltrato doméstico en las mujeres y en sus hijos e hijas. Jornada de Trabajo para el profesorado. Proyecto NAHIKO. EMAKUNDE; 2004.

19. Cantón Duarte J, Cortés Arboleda M R. Effects of child maltreatment on aggressive and withdrawn behaviour. International Journal of Child and Family Welfare 1997;2:190-205.

20. Sepúlveda A, Sepúlveda P. Consecuencias de la violencia para las mujeres. Proyecto Daphne. Europa Rosa. Roma. 1999.

2I. Echeburúa E. Superar un trauma. El tratamiento de las víctimas de sucesos violentos. Editorial Pirámide. Madrid 2004. Pp:48-79.

22. American Psychiatric Association (1994). Manual diagnostico y estadístico de los trastornos mentales DSM-IV. Madrid: Masson.

23. Margolin G, Gordis EB. The Effects of family and community violence on children. Annual Review of Psychology 2000;51:445479.

24. García-Moreno, C. Violencia contra la mujer. Género y equidad en la salud. Organización Panamericana de la Salud y Harvard Center for Population and Development Studies; 2000 (consultado 05/II/2003). Disponible en: http://www.paho.org/ Spanish/DBI/p006.htm.

25. Salas Bahamón LM. Transmisión Intergeneracional de La Violencia Intrafamiliar: evidencia para las familias colombianas. Documento CEDE 2005-47; ISSN I657-719I (edición electrónica). 26. Save the Children \& Equipo de Investigación Márgenes y Vínculos (2006). Atención a los Niños y Niñas Víctimas de Violencia de Género en Andalucía. Junta de Andalucía. 